\title{
Avaliação da atenção no contexto do trânsito: uma amostra da Bahia - Brasil
}

\author{
*Marlene Alves da Silva, **Luís Sérgio Sardinha, *** Leonardo Augusto Couto Finelli \\ *ABCtran- Associação Bahiana de Clínicas de trânsito e Clínica Fênix - Vitória da Conquista - BA. **Universidade Anhanguera de \\ São Paulo e Universidade Braz Cubas ***FUNORTE - Faculdades Integradas do Norte de Minas e FASI - Faculdade de Saúde \\ Ibituruna
}

\section{Resumo}

No Brasil, um teste psicológico, para ser considerado em condições de uso, deve ser aprovado pelo Conselho Federal de Psicologia, que regulamenta a matéria por meio da Resolução 002/2003. Resolução apoiada em várias publicações internacionais. $\mathrm{O}$ objetivo deste trabalho foi buscar evidência de validade para os testes de Atenção Dividida e Alternada. A amostra foi composta 311 pessoas com idade entre 18 a 68 anos de uma região do nordeste brasileiro. Foram calculadas a correlação de Pearson e a ANOVA entre os totais de pontos. Os resultados apontam que ocorreu correlação moderada, positiva e significativa entre as pontuações dos testes. Os dados disponíveis permitem concluir que, nesta amostra, existem evidências de validade dos referidos testes.

Palavras chave: atenção concentrada, evidencias de validade, trânsito.

No Brasil, um teste psicológico, para ser considerado em condições de uso, deve ser aprovado pelo Conselho Federal de Psicologia, que regulamenta a matéria por meio da Resolução 002/2003 (CFP, 2003). Tal resolução dispõe sobre elaboração, comercialização e uso dos testes psicológicos. Para isto devem ser apresentados estudos atualizados relativos aos parâmetros psicométricos do teste, particularmente os que evidenciem sua validade e precisão, pelos profissionais que fazem uso dos mesmos.

A validade diz respeito ao aspecto da medida de ser congruente com a propriedade medida, nesse caso a atenção; assim, pode-se dizer que um teste é válido quando ele mede o que se propõe a medir (Pasquali, 2003, Urbina, 2007). Para isso faz necessário tal construto estar ancorado em uma teoria e testado empiricamente. Dessa forma, a validade de um instrumento de avaliação deve ser apoiada por uma análise complexa, relacionada com fatores como objetivos, contexto, variáveis a serem examinadas, sujeito ou população e, consequentemente, os resultados e propósitos do processo avaliativo.

Em decorrência desta regulamentação existe um grande número de instrumentos que são estudados com o objetivo de atualizar e aperfeiçoar as avaliações já realizadas. Dada à importância dos testes psicológicos no processo de avaliação psicológica, conforme determinam as Resoluções do Conselho Federal de Psicologia (CFP, 2003; 2009; 2011), entre outras, essa revisão deverá ser realizada pelo autor/detentor dos direitos de comercialização do teste, que deve nomear psicólogo técnico responsável pela edição do instrumento, assim como por pesquisadores que deverão publicar os estudos nos veículos de comunicação científica.

As pesquisas de padronização devem considerar um sistema único de aplicação para todo o Brasil. Não obstante, esse é um país de dimensões continentais, e assim as pesquisas que consideram as normas, com critérios para interpretação, por exemplo, tabelas, para a correção dos testes, devem atender a aspectos das diversas regiões do país. Nesse sentido, faz-se mister que tais pesquisas considerem tal diversidade. Além disso, a Resolução 006/2009 (CFP, 2009) também considera que tais pesquisas devem ser renovadas periodicamente, não podendo exceder espaço temporal de 15 anos antes de novas atualizações.

Considerando a diversidade de instrumentos de avaliação psicológica reconhecidos como adequados para uso no Brasil, tem-se que é possível aferir várias dimensões psíquicas (ou traços latentes). Uma dessas dimensões é a atenção, que pode ser entendida como a capacidade de focar as funções executivas em uma dada tarefa, ou estímulo, em detrimento de outras (Finelli, Abreu, 2013). São vários os instrumentos psicológicos que medem atenção, dentre eles, o Teste de Atenção Dividida (TEADI) e Teste de Atenção Alternada (TEALT) editados por Rueda (2010). Esses fornecem medidas referentes à capacidade da pessoa procurar mais de dois estímulos simultaneamente e de alternar a atenção, ora focalizando em um estímulo e ora em outro estímulo. No manual do teste encontram-se estudos com uma população adulta de 18 a 72 anos, estudantes universitários e pessoas que procuraram a avaliação psicológica para à obtenção, adição, renovação ou mudança de categoria da Carteira Nacional de Habilitação - CNH.

$\mathrm{Na}$ primeira edição do manual, constam tabelas normativas para duas cidades brasileiras, sendo que as normas para o estado de Sergipe teve sua amostra composta por estudantes universitários; já para a Bahia, o grupo amostral foi composto por candidatos que realizaram avaliação psicológica no contexto do trânsito. No tocante as normas baiana, teve a participação da coleta de dados da primeira autora desse manuscrito, e a amostra constou de 479 pessoas para o TEADI e de 442 candidatos para o TEALT. Posteriormente, foi publicado um compêndio complementar ao manual, com dados normativos de sete cidades do estado do Paraná, com uma amostra de 818 candidatos a $\mathrm{CNH}$; sete cidades do estado de Santa Catarina, com uma amostra de 437 candidatos a $\mathrm{CNH}$; e três cidades de São Paulo, com amostras de 205 
candidatos a CNH e 35 universitários (Santana e cols., 2011).

No caso da avaliação psicológica na área do trânsito, essa é compulsória e determinada pelo Conselho Nacional de Trânsito (CONTRAN) e Conselho Federal de Psicologia (CFP). O CFP em 2011, conforme nota técnica $\mathrm{n}^{\circ} 1$, ressaltou a necessidade de que ao realizar avaliação psicológica no contexto do trânsito sejam aferidos, no mínimo, três tipos de atenção. Ainda, o profissional deverá pesquisar dentre os testes psicológicos, aprovados para uso, os que melhor atendem aos propósitos da avaliação.

Considerando o exposto, esse estudo contempla a busca de evidência de validade para os testes de Atenção Dividida e Alternada, que avaliam dois tipos de atenção e atualizar as tabelas normativas para uma região da Bahia - Brasil.

\section{Método}

A pesquisa assumiu caráter transversal, retrospectiva, e documental, valendo-se da análise de prontuários de candidatos a $\mathrm{CNH}$ e motoristas que buscavam adição, renovação ou mudança de categoria da $\mathrm{CNH}$, avaliados em uma clínica da cidade de Vitória da Conquista, localizada à região sudoeste no estado da Bahia - Brasil. As avaliações psicológicas foram realizadas no período de setembro de 2014 a março de 2015. Os resultados dos testes foram transpostos para planilha do software SPSS Statistical Package for Social Sciences (versão 13.0) para a realização das análises estatísticas.

\section{Instrumentos}

O Teste de Atenção Dividida (TEADI) fornece uma medida sobre a capacidade do indivíduo para dividir a atenção, de buscar mais de dois estímulos simultaneamente. Já o Teste de Atenção Alternada (TEALT) avalia a capacidade que o sujeito tem para focar a atenção de modo alternado em distintos estímulos. Nos dois testes, ao buscar os estímulos alvejados, o indivíduo deve desconsiderar os estímulos que dificultem esta ação. Os dois instrumentos podem ser aplicados individual ou coletivamente. A forma de avaliação é realizada por um crivo (cada teste tem o seu), onde obtém-se o total de acertos (A), erros (E) e omissões (O). Para o cálculo do Total de Pontos (TP) aplica-se a fórmula $\mathrm{TP}=\mathrm{A}-(\mathrm{E}+\mathrm{O})$. A editora oferece também um sistema de correção informatizada (Rueda, 2010).

Considerando as evidências de validade para o TEADI, quanto ao construto a avaliação da análise de variância $\quad($ ANOVA $F \quad(2,864)=53,57, p<0,001)$ indicou a existência de três grupos etários, reconhecendo que os níveis de atenção decaem com a idade. Com relação a critério, foi verificada com a escolaridade constatando dois grupos (ANOVA $F$ $(6,477)=17,16, p<0,001)$; e ainda, de critério com o Teste de Atenção Dividida - AD cujas correlações de Pearson, para as diversas faixas etárias foram sempre estatisticamente significativas com magnitudes moderadas e positivas (variando de 0,44 a 0,66) (Rueda, 2010).

Já as evidências de validade para o TEALT, quanto ao construto a avaliação da Análise de Variância (ANOVA $F(2,797)=60,01 p<0,001)$ indicou a existência de três grupos etários, reconhecendo que os níveis de atenção decaem com a idade. Esses foram pouco diferentes dos intervalos etários verificados para o TEADI. Com relação a critério, foi verificada com a escolaridade constatando três grupos (ANOVA $F$ $(5,475)=19,09, p<0,001)$; e ainda, de critério com o Teste de Atenção Concentrada - TEACO - FF cujas correlações de Pearson, para as diversas faixas etárias foram sempre estatisticamente significativas com magnitudes moderadas e positivas (variando de 0,35 a 0,68) (Rueda, 2010).

Quanto as evidências de precisão o TEADI apresentou coeficientes de alfa de Cronbach e pelos métodos das metades de Guttman e Spearman-Brown variando de 0,83 a 0,89 para as diversas faixas etárias e população geral. Já para o TEALT as mesmas medidas variaram entre 0,81 a 0,87 (Rueda, 2010).

\section{Resultados e discussão}

A amostra constituiu-se de 311 prontuários com os dados do teste TEADI e TEALT. Desses 237 (76,2\%) são homens e 74 (23,8\%) mulheres, sendo 165 (53,1\%) solteiros, 137 (44\%) casados ou em convivência estável e 09 (2,9\%) separados, divorciados ou viúvos. Quanto à escolaridade 96 (30,9\%) estudaram até o ensino fundamental, $165(53,1 \%)$ o ensino médio incompleto e completo e 49 (16\%) o ensino superior incompleto a pós-graduação. A idade variou de 18 a 68 anos (média de idade $=29,79$ anos, com DP $=9,6$ anos, mediana $=$ 28 anos e a moda $=18$ anos), conforme pode ser observado na Figura 1.

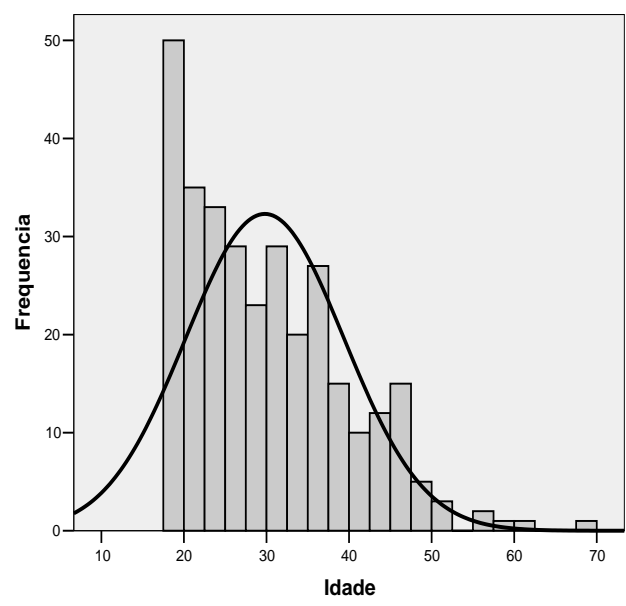

Figura 1 - Distribuição de idades

Observa-se que a maioria $50,8 \%$ da amostra concentra-se entre a idade de 18 a 28 anos, desses 
27,3\% tem idade até 22 anos. Ressalta que no Brasil a idade mínima para requerer a primeira habilitação é de18 anos. Quanto à escolaridade, essa variou de $1^{\circ}$ ano do ensino fundamental a ensino superior completo.

A média de pontos na medida do Teste de Atenção Dividida (TEADI) foi de 102,23, com desvio padrão de 37,3; sendo a pontuação mínima de zero e a máxima de 308 pontos. Já no Teste de Atenção Alternada (TEALT) a média foi de $77,22(D P=29,28)$, com uma pontuação mínima foi zero e uma máxima de 158 pontos.

Para verificar as possíveis relações entre ambos os testes, realizou-se uma correlação de Pearson entre os totais de pontos, cujo resultado forneceu um $r=0,464 \mathrm{e}$ $p=0,001$. O resultado apontou que ocorreu correlação moderada, positiva e significativa entre as pontuações dos testes TEADI e TEALT.

Quanto à análise das distribuições das produções em ambos os testes, produção dos histogramas assemelhamse a curvas normais, o que possibilita a investigação da distribuição percentílica.

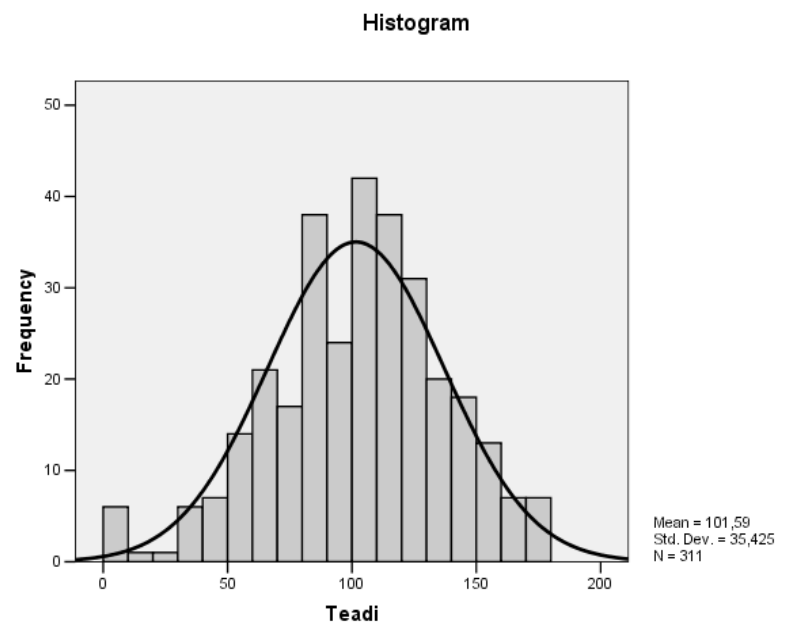

Figura 2 - Distribuição de pontuações no TEADI

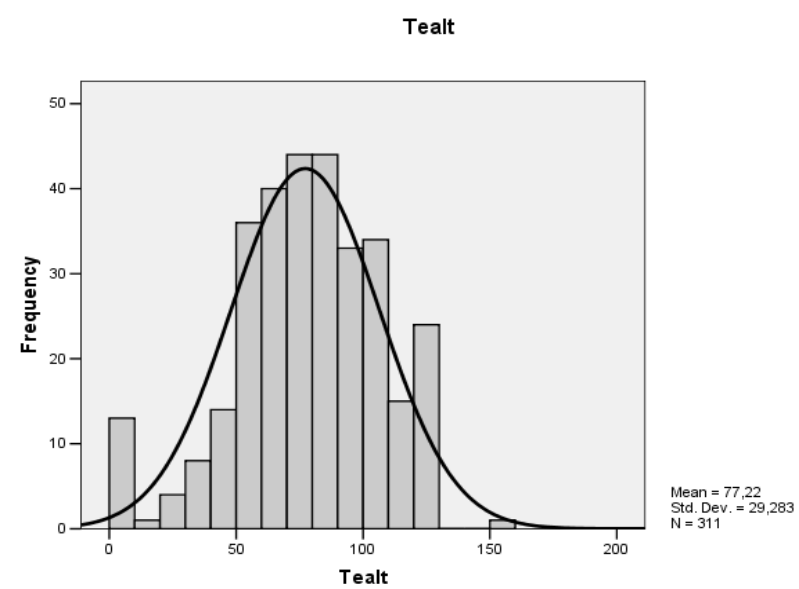

Figura 3 - Distribuição de pontuações no TEALT
De modo a confirmar tal hipótese, foi realizado o teste de normalidade de Kolmogorov-Smirnov (KSZ) ), que é um teste de análise paramétrica que assume que a distribuição dos dados é uma distribuição normal a priori. O escore do KSZ não é importante em si, mas o nível de significância (p) a ele atribuído indica que se $p$ for estatisticamente significativo ( $<=0,05)$ a distribuição em questão será significativamente diferente de uma distribuição normal. Por outro lado, de forma similar se $p$ não for estatisticamente significativo ( $p>0,05)$ o KSZ nos informa que os dados não diferem siginificativamente de uma distribuição normal, ou seja, aquela distribuição pode ser considerada normal (Siegel; Castellan, 1988).

A análise realizada encontrou $\mathrm{KSZ}=0,825 ; \mathrm{p}=0,505$ para os dados do TEADI e KSZ $=0,841 ; \mathrm{p}=0,479$ para os dados do TEALT, o que indica que a distribuição dos dados analisados não diferem de modo estatisticamente significativo de uma distribuição normal.

A partir de tais dados foi calculada a distribuição de frequência considerando a pontuação percentílica considerando os decis, os quartis e escores extremados $(\mathrm{P}=5$ e $\mathrm{P}=95)$ que são apresentados na tabela 1 .

Tabela 1:

Análise de frequência da pontuação dos testes e distribuição percentílica

\begin{tabular}{|c|c|c|c|c|c|}
\hline & \multicolumn{2}{|c|}{$\begin{array}{l}\text { Dados da } \\
\text { pesquisa }\end{array}$} & \multicolumn{2}{|c|}{$\begin{array}{l}\text { Dados do } \\
\text { manual }\end{array}$} \\
\hline & & TEADI & TEALT & TEADI & TEALT \\
\hline $\mathrm{N}$ & & 311 & 311 & 865 & 798 \\
\hline Média & & 101,59 & 77,22 & 108,61 & 92,52 \\
\hline Mediana & & 104,00 & 78,00 & 113 & 103 \\
\hline Moda & & 81 & 0 & 128 & 126 \\
\hline Desvio padrão & & 35,425 & 29,283 & $\begin{array}{r}43,6 \\
2\end{array}$ & $\begin{array}{r}41,4 \\
9\end{array}$ \\
\hline Mínimo & & 0 & 0 & -126 & -136 \\
\hline Máximo & & 176 & 158 & 180 & 128 \\
\hline Percentil & 5 & 44,0 & 20,00 & - & - \\
\hline & 10 & 55,4 & 43,00 & 50 & 52 \\
\hline & 20 & 71,4 & 56,00 & 75 & 72 \\
\hline & 25 & 81,0 & 60,00 & 84 & 79 \\
\hline & 30 & 84,0 & 62,00 & 92 & 84 \\
\hline & 40 & 95,0 & 71,00 & 103 & 95 \\
\hline & 50 & 104,0 & 78,00 & 113 & 103 \\
\hline & 60 & 112,0 & 85,00 & 124 & 111 \\
\hline & 70 & 120,4 & 94,00 & 134 & 118 \\
\hline & 75 & 125,0 & 98,00 & 140 & 121 \\
\hline & 80 & 131,6 & 104,0 & 146 & 123 \\
\hline & 90 & 146,0 & 113,80 & 161 & 126 \\
\hline & 95 & 156,0 & 123,00 & - & - \\
\hline
\end{tabular}

Tal quando comparada com os dados para população geral disponíveis no manual apresenta diferenças marcantes. Assim aplicou-se o teste $t$ de Student de modo a verificar se tais dados diferiam de modo estatisticamente significativos dos disponíveis no manual. Como os resultados verificados apresentavam 
nível de significância $p=0,001$ para o TEADI e $p<$ 0,001 para o TEALT, pode-se concluir pela necessidade da apresentação dessa nova tabela considerando dados que compreendem aspectos da regionalidade.

Comparando-se os desempenhos entre os testes TEADI e TEALT, verifica-se uma correlação moderada e positiva $(r=0,48 ; p=0,000)$ entre a atenção dividida e alternada. Tal dado indica a relação entre as formas distintas de atenção, sem deixar de contemplar que tratam-se de dimensões diferentes de um mesmo construto.

\section{Conclusão}

Da análise dos dados dessa amostra e informações apuradas, constata-se evidência empírica da correlação entre a atenção dividida e alternada. Esse conhecimento pode contribuir para a continuidade das pesquisas envolvendo outros contextos, bem como apoiar as decisões tomadas na avaliação da atenção na área do trânsito.

Os resultados indicaram também que a distribuição percentílica verificada para a população pesquisada diferiu de modo estatisticamente significativo dos dados da pesquisa inicial disponíveis no manual do teste. Tal informação indica a necessidade de publicação dessas tabelas, assim como de realização de novas pesquisas que considerem aspectos de regionalidade dos grupos amostrais.

\section{Referências}

Conselho Federal de Psicologia (2003) Resolução CFP $n^{\circ} 02 / 2003$. Disponível na Word Wide Web: http://www.pol.org.br. Obtido em 10 de junho de 2015.

Conselho Federal de Psicologia (2009) Resolução CFP n06/2009. Disponível na Word Wide Web: http://www.pol.org.br. Obtido em 10 de junho de 2015.

Conselho Federal de Psicologia (2011) Nota Técnica $n^{\circ} 001 / 2011$. Disponível na Word Wide Web: http://www.pol.org.br. Obtido em 10 de junho de 2015.

Finelli, L. A. C., \& Abreu, A. C. C. (2013) Atenção concentrada, instrumentos dispersos: questionando o constructo a partir das pesquisas de validação do Teste AC. Monografia, Departamento de Psicologia, Faculdade de Saúde Ibituruna - FASI, Montes Claros, Brasil.

Pasquali, L. (2003) Psicometria: teoria dos testes na psicologia e na educação. Petrópolis: Editora Vozes.

Rueda, F. J. M. (2010). Teste de Atenção Dividida (TEADI) e Teste de Atenção Alternada (TEALTI). São Paulo: Casa do Psicólogo.

Siegel, S., \& Castellan, N.J. (1988). Nonparametric statistics for the behavioral sciences (2nd Ed.). New York, NY: McGraw-Hill.

Urbina, S. (2007). Fundamentos da Testagem Psicológica. Porto Alegre: Artmed 